\title{
Attention-Driven Eye Gaze And Blinking For Virtual Humans
}

\author{
Christopher Peters \\ Image Synthesis Group \\ Department of Computer Science \\ Trinity College \\ Dublin 2, Ireland \\ Christopher.Peters@cs.tcd.ie
}

\author{
Carol O' Sullivan \\ Image Synthesis Group \\ Department of Computer Science \\ Trinity College \\ Dublin 2, Ireland \\ Carol.OSullivan@cs.tcd.ie
}

Gaze and blinking have recently been considered for application to social interactions among computer characters based on emotional states. In this sketch, we present improvements to a gaze controller in terms of both the contributions by the joints involved in the motion and also by a closer analysis of the role of blinking and blink synchronisation in the realism of the final animation. Unlike previous approaches, our controllers are driven by internal character states derived from, among other factors, a model of visual attention and short-term memory. Given a position to look at and taking into account internal state information such as an agent's interests, motivation and memory, the gaze and blink controllers are charged with the responsibility of executing an appropriate gaze motion in an expressive manner.
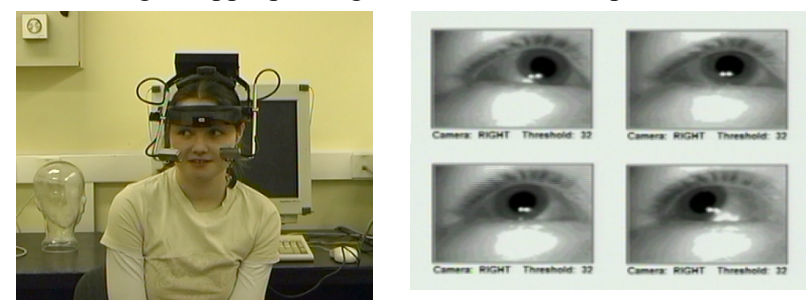

Figure 1. Gaze evoked blink experiments (left) and resulting eye movement video (right).

We have previously presented a system [Peters and O' Sullivan 2003], based on research from the field of cognitive engineering [Itti et al. 1998], for the automatic generation of bottom-up visual attention behaviours in virtual humans. The system processes an input image from a synthetic vision module, calculating local contrast for intensity, orientation and colour features and then combining the results into a single saliency map using a normalisation procedure. The output of the attention system is passed to a gaze controller, in order to generate a realistic gaze motion towards the current most salient location.

Gaze motions are parameterised in terms of the contribution of the eye, head, neck and shoulder joints to the movement and the total dwell time spent on the target before moving to the next target. Based on evidence from psychology [Bard et al. 1991], virtual humans are tagged with a head-move attribute, defining how likely they are to turn their heads to look at objects.

Although subtle, we regard blinking motions as being highly important for conveying realistic gaze motions. Our primary focus is on the relationship between blinking and gaze shifts, referred to as gaze-evoked blinks in the psychology literature [Evinger et al. 1994]. These blinks typically begin simultaneously with the initiation of the head/eye movement and the probability of a blink increases with the size of the gaze shift. Furthermore, the amplitude of the gaze motion has been shown to affect the magnitude of the blink. These features have been incorporated into our gaze control system; when a gaze shift is imminent, a blink procedure is invoked and initialises a blink animation with the desired blink magnitude for the corresponding eccentricity.

A number of preliminary experiments were conducted on three test subjects to examine both the timing and nature of gaze evoked blinking for varying eccentricities in human subjects (Figure 1). An S.M.I. Eyelink eye-tracker was used to obtain close-up images of the eye during gaze motions. We also generated a number of animations where a virtual actor was given the task of looking at targets located at similar eccentricities to those in the experiments (Figure 2).

\section{References}

Peters, C. AND O' Sullivan, C. 2003. Bottom-up visual attention for virtual human animation. Computer Animation and Social Agents 2003, in press.

ItTI, L., Koch, C., AND NieBuR, E. 1998. A model of saliencybased visual attention for rapid scene analysis, California Institute of Technology, $\mathrm{PhD}$ Thesis.

Bard, C., Fleury, M. AND PAillard, J. 1991. Different patterns in aiming accuracy for head-movers and non-head movers. In: A.Berthoz, W.Graf \& P.P.Vidal (eds). The Head-neck Sensorymotor System. Oxford University Press, Oxford, pp 582-586.

Evinger, C., Manning, K., Pellegrini, J., Basso, M., Powers, A., AND SiBONy, P. 1994. Not looking while leaping: the linkage of blinking and saccadic gaze shifts. Experimental Brain Research, 100, pp 337-344.
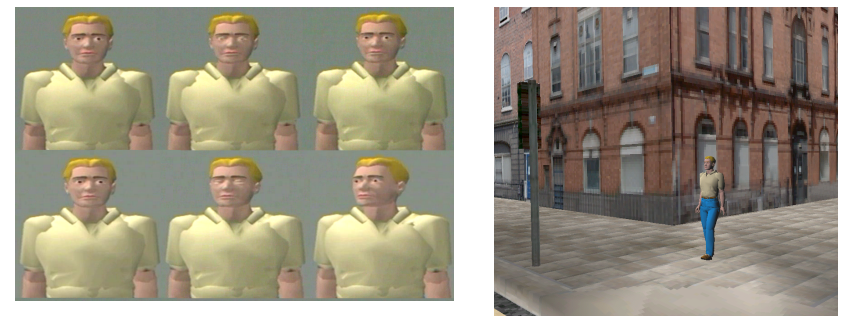

Figure 2. Animation frames from the gaze generator for three different eccentricities (left). Gaze generator at work in a virtual city environment (right). 